\title{
Diskushernie immer operieren?
}

\author{
Swiss Medical Board
}

Das Swiss Medical Board hat in seinem neusten Bericht untersucht, ob eine Diskushernie immer operiert werden soll. Es kommt zum Schluss, dass in der akuten Phase, sofern keine funktionell relevanten, motorischen Ausfälle und Beschwerden vorliegen, die konservative Behandlung einer Operation vorzuziehen ist. Wenn Erfolge nach ca. drei Monaten ausbleiben oder sich der Zustand verschlechtert, sollte ein chirurgischer Eingriff in Betracht gezogen, der Patient sorgfältig aufgeklärt werden.

Das Swiss Medical Board (SMB) hat Organisation und Prozesse weiterentwickelt Der vorliegende Bericht wurde im Rahmen der neuen Organisation und mit weiterentwickelten Prozessen erstellt. Die operativen Gremien des SMB sind seit 2014 organisatorisch getrennt in ein Assessment Team und ein Appraisal Komitee. Erstmals wurde für den vorliegenden Bericht auch der Stakeholder-Prozess erweitert und vor dem Appraisal ein StakeholderHearing durchgeführt. Die Stakeholder-Kommentare sind weit möglichst in den Appraisal-Bericht eingeflossen und liegen dem Appraisal-Bericht vollständig bei.
Rückenschmerzen sind sehr häufig und haben unterschiedliche Ursachen. In vielen Fällen sind sie auf Bandscheibenvorfälle, genannt Diskushernien, zurückzuführen. Diskushernien können durch Druck auf die seitlich der Wirbelsäule austretenden Rückenmarksnerven zu deren Reizungen oder Schädigungen (Radikulopathie) führen. Die damit verbundenen Symptome reichen von Schmerzen im Bein bis zu Gefühlsstörungen (sensiblen Ausfällen) und Muskellähmungen (motorischen Ausfällen).

Radikulopathien können konservativ oder operativ behandelt werden. In der Frühphase kommt meist eine konservative Behandlung zum Einsatz. Dabei geht es primär um Schmerzlinderung. Bei leichten Beschwerden ist dies in der Regel ausreichend. Bei funktionell relevanten motorischen Ausfällen ist dagegen meist eine operative Behandlung angezeigt. In vielen Situationen ist jedoch unklar, welches die am besten geeignete Behandlung ist. Hier setzt die Fragestellung des Berichts «Akute oder subakute lumbale Radikulopathien wegen Diskushernien: konservative versus operative Behandlung» an.

\section{Empfehlungen zur Therapie und Hinweise zur Forschungstätigkeit}

Ein unabhängiges universitäres Team arbeitete Daten zur medizinischen Evidenz auf und stellte sie im sogenannten Assessment-Bericht dar. Eine Expertengruppe holte Stellungnahmen von betroffenen Partnern (medizinischen Fachgesellschaften, Therapeuten, Finanzierern, Patienten) ein und hielt darauf basierend Abwägungen und Empfehlungen im Appraisal-Bericht fest. Dabei wurden die in den Konsultationen geäusserten Meinungen weit möglichst in die Schlussfolgerungen einbezogen.

Die Datenlage zur Fragestellung ist für beide Therapieformen lückenhaft und älteren Datums. Daten zu wesentlichen Entwicklungen sind weder für die kon- servative noch für die operative Therapie vorhanden. Umso wichtiger ist, dass Informationen aus der Praxis zu den aktuell in der Schweiz angewendeten Standards in die Betrachtungen miteinbezogen werden konnten. Das Expertengremium hat unter Berücksichtigung des Assessment-Berichts und der Kommentare betroffener Leistungserbringer folgende Empfehlungen formuliert:

- Patienten mit akuten (<6 Wochen) oder subakuten ( $<12$ Wochen) lumbalen Radikulopathien wegen Diskushernien, ohne funktionell relevante, motorische Ausfälle und Beschwerden sind im Rahmen eines interdisziplinären Therapieansatzes zu behandeln, wobei einer konservativen Behandlung Priorität einzuräumen ist.

- Die Patienten sind mit geeigneten Mitteln ausführlich über die jeweiligen Vor- und Nachteile, insbesondere auch die Art und Häufigkeit möglicher unerwünschter Wirkungen der verschiedenen Behandlungsansätze aufzuklären.

- Im Hinblick auf die ungenügende Evidenzlage sind in der Schweiz Forschungsanstrengungen in den folgenden vier Bereichen anzustossen, zu finanzieren und durchzuführen:

1. Eine adäquat grosse, randomisierte Studie, welche den primär operativen mit dem primär konservativen Ansatz auf der Basis der heutigen Behandlungsmodalitäten vergleicht.

2. Randomisierte Vergleichsstudien zur Untersuchung der erwünschten und der unerwünschten Wirkungen des breiten Spektrums unterschiedlicher konservativer Behandlungen.

3. Erforschung von Prognose-Kriterien für die Erkennung jener Patientengruppen, die von einer frühzeitigen Operation profitieren.

4. Bessere Dokumentation der jeweiligen Langzeitverläufe nach primär konservativem und primär operativem Therapieansatz.

Der Assessment- und der Appraisal-Bericht sind auf der Webseite des Swiss Medical Board veröffentlicht. 